\title{
Harnessing the Power of the Crowd for Bioimage Analysis
}

Martin Jones1, Harry Songhurst1,2, Chris Peddie1, Anne Weston1, Helen Spiers3, Chris Lintott3 and Lucy M. Collinson1*

1. Electron Microscopy Science Technology Platform, The Francis Crick Institute, London, UK

2. University of Manchester, Manchester, UK

3. Zooniverse, University of Oxford, Oxford, UK

* Corresponding author: lucy.collinson@crick.ac.uk

Many different imaging modalities now routinely produce huge amounts of data thanks to increased acquisition speeds and extensive automation. Whilst computational image analysis techniques are constantly improving, it is often necessary to use manual analysis to some degree, in particular to provide ground truth annotations in order to train machine learning systems such as convolutional neural networks.

Volume electron microscopy techniques, such as serial block face SEM (SBF SEM), focused ion beam SEM (FIB SEM) and array tomography (AT) [1], produce datasets in the terabyte regime. In many cases, much of the analysis is still performed manually, which represents a major bottleneck in the workflow, restricting the amount of information that can be extracted from these rich datasets. Furthermore, single manual annotations may be prone to subjectivity that is not meaningfully encoded in the binary segmentation. To attempt to address these problems, we collected benchmark volume EM data from resinembedded HeLa cells [2], and built a citizen science platform 'Etch a Cell' [3] on the Zooniverse platform [4] to procure segmentations from a distributed set of volunteers. So far, the project has over 5000 contributors from around the world, with over 100,000 individual segmentations. By aggregating several non-expert contributions per image, we are able to reconstruct expert-level segmentations with additional information about the confidence of each pixel identification. The project will provide a set of general tools that will enable other labs to incorporate crowdsourcing into their workflows for segmentation and other types of image analysis.

Beyond the research applications, we have also found the project to be an effective outreach and education tool, letting students and non-experts gain an insight into the scientific process at the raw data level [5].

\section{References:}

[1] CJ Peddie and LM Collinson, Micron 61 (2014), p. 9.

[2] EMPIAR, https://www.ebi.ac.uk/pdbe/emdb/empiar/entry/10094/ (accessed March 6th, 2019). https:/www.ebi.ac.uk/pdbe/emdb/empiar/entry/10106/ (accessed March 6th, 2019).

[3] Etch a Cell, tinyurl.com/etchacell (accessed March 6th, 2019).

[4] C Lintott et al., Mon.Not.Roy.Astron.Soc. 389 (2008), p. 1179.

[5] The authors acknowledge funding from The Francis Crick Institute which receives its core funding from Cancer Research UK (FC0010004), the UK Medical Research Council (FC0010004), and the Wellcome Trust (FC0010004). 


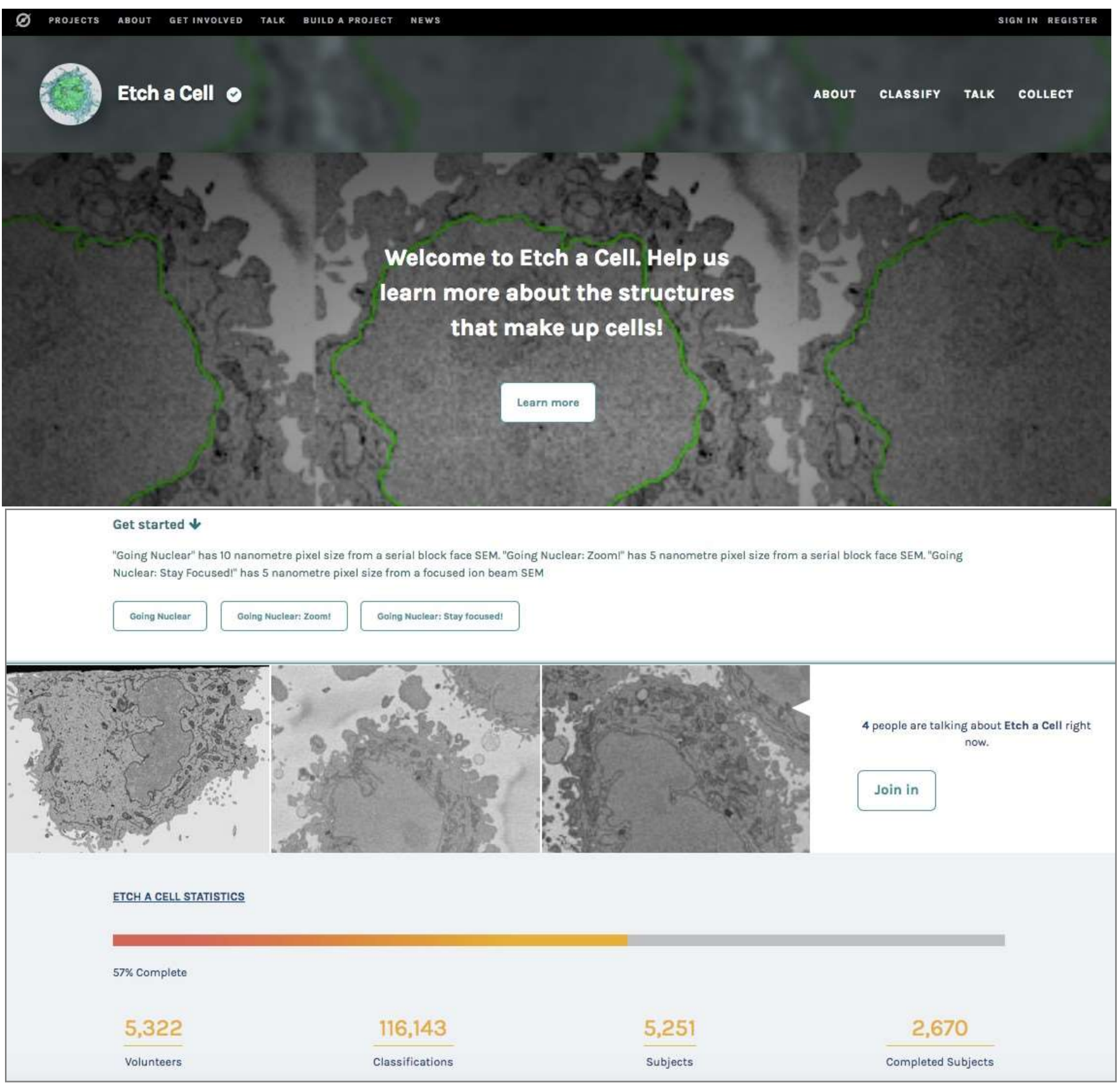

Figure 1. The Etch a Cell interface on the Zooniverse platform, which currently hosts three projects whose aim is to segment the nuclear envelope from HeLa cells imaged using SBF SEM and FIB SEM. 